\title{
A música como auxílio no tratamento fisioterapêutico em pacientes com autismo: estudo de caso
}

\author{
The music as an aid in physiotherapy \\ treatment in patients with autism: $a$ \\ case estudy
}

FisiSenectus . Unochapecó Ano 3, n. 2 - Jul/Dez. 2015 p. 3-11

Nelson Guilherme Trindade. nelsontrindadengt@gmail.com

Fisioterapeuta. Cesufoz - Centro de Ensino Superior de Foz do Iguaçu.

Everson Prestes. eversonprestes1@gmail.com

Fisioterapeuta. Cesufoz - Centro de Ensino Superior de Foz do Iguaçu.

Nayara Correa Farias. ncf.fisio@gmail.com

Fisioterapeuta, Mestre. Cesufoz - Centro de Ensino Superior de Foz do Iguaçu.

\section{Resumo}

Introdução: A música pode ser utilizada como meio de intervenção em déficits sociais, comunicativos e cognitivos em indivíduos com autismo, promovendo muitos benefícios quando associada à fisioterapia. Objetivos: Avaliar e promover ganhos nos aspectos afetivos, cognitivo e motor. Materiais e métodos: A presente pesquisa caracteriza-se por ser um estudo quanti e qualitativo, utilizando uma amostra de três crianças com autismo, com idades entre cinco a sete anos. Utilizou-se a avaliação diagnóstica Childhood Autism Rating Scale (CARS) pré e pós-intervenção e foi desenvolvido um protocolo de condutas fisioterapêuticas com o auxílio da música em um período de dois meses. Resultados: Verificou-se, por meio das observações dos vídeos e em sala de aula, evolução no aprendizado cognitivo, motor e funcional em todos os indivíduos. No entanto, de acordo com a pontuação CARS, não houve evolução significativa. Conclusão: Identificaramse ganhos cognitivos, motores e de interação social no grupo estudado. A partir do estudo foi possível perceber, também, a necessidade de um maior período de intervenção e instrumentos de avaliações diagnósticas mais precisas e sensíveis para essa população.

\section{Palavras-chave}

Estudos de casos. Transtorno autístico. Música. Fisioterapia.

\section{Fisißenectus}




\begin{abstract}
Introduction: The song has been used as an intervention in social, communicative and cognitive deficits in individuals with autism, providing many benefits when combined with physiotherapy. Objective: To evaluate and promote gains in the affective, cognitive and motor aspects. Materials and methods: It is characterized by a quantitative and qualitative study, through a screening, selecting three children with autism, five to seven years. Was used the diagnostic evaluation Childhood Autism Rating Scale - (CARS), before and after -intervention and conducting physical therapy procedures with music assistance through an established protocol in a period of two months. Results: It was found through observations of videos and in the classroom, progress in cognitive, motor, and functional learning in all subjects. According to the CARS score, there were no significant evolution. Conclusion: Were identified cognitive, motor and social interaction gains in the study group. From the study it was possible to realize also the necessity of a longer period of intervention tools and diagnostic assessments more accurate and sensitive for this population.
\end{abstract}

\title{
Keywords
}

Case studies. Autistic disorder. Music. Physical therapy specialty.

\section{Introdução}

$\infty \times \infty \times \infty \times \infty \times \infty \times \infty \times \infty \times \infty \times \infty \times \infty \times \infty \times \infty \times \infty)$

A ocorrência de autismo aumentou significativamente em 1990 e no início de 2000, ${ }^{1}$ apresentando prevalência no sexo masculino, em irmãos de crianças com Transtorno do Espectro Autista (TEA) e, ainda, em indivíduos com outros distúrbios do desenvolvimento. O aumento da ocorrência deve-se, parcialmente, às vigilâncias por diagnósticos mais eficazes na avaliação do TEA $^{2}$

A etiologia do autismo é desconhecida e complexa, provendo de distúrbios heterogênicos, diagnosticados subjetivamente na base de um grande número de critérios, seguindo os comprometimentos ocorridos pelo Transtorno Invasivo do Desenvolvimento (TID) ${ }^{2}$.

Conforme a Diagnostic and Statistical Manual of Mental Disorders (DSM-IV), ${ }^{3}$ o TEA é caracterizado pela presença de um desenvolvimento acentuadamente anormal na interação social e comunicação, restringindo-se a um repertório de atividades e interesses. As manifestações do transtorno variam dependendo do nível de desenvolvimento e da idade cronológica do indivíduo.

Apesar de serem fisicamente normais para a idade cronológica, os déficits cognitivos, motores e sociais podem ser observados antes dos três anos de idade, sendo que cerca de $50 \%$ possuem quociente de inteligência (QI) inferior a $50^{4}$.

Suas características, como a inflexibilidade e a dificuldade de relacionamento interpessoal, podem ser explicadas por um comprometimento funcional na região lobo cerebral frontal ${ }^{5}$. Apresentando déficits de linguagem e comunicação ${ }^{6}$, podem estar associadas à dificuldade motora, à redução do tônus muscular, ao equilíbrio, à coordenação e à cognição ${ }^{7}$.

A música tem sido utilizada como meio de intervenção em déficits sociais, comunicativos e cognitivos nos indivíduos com autismo ${ }^{8}$. Visto que, a linguagem musical baseia-se nos ritmos e percussões, gerando estados de atenção conjunta entre o terapeuta e o paciente ${ }^{9}$, favorecendo a percepção dos diferentes estímulos para gerar movimentos, ação e linguagem ${ }^{10,11}$.

Esse recurso constitui, por meio de diversas teorias, um campo de pesquisa altamente promissor para a área da saúde, orientando práticas com resultados significativos no tratamento de patologias que afetam a capacidade física, cognitiva ou subjetiva dos indivíduos ${ }^{12}$, devido ao seu importante papel na formação neural, motora e demais expressões ${ }^{10}$ e promovendo a inclusão de uma equipe multidisciplinar, juntamente com outras áreas de atuação terapêutica, inclusive a fisioterapia . 
A abordagem fisioterapêutica no paciente com autismo tem por objetivo concentrar-se nos comprometimentos motores que causam limitações funcionais e no aprendizado cognitivo de tarefas funcionais, pois, a estimulação de uma tarefa, emerge de um processo de autoorganização a adaptação do Sistema Nervoso Central (SNC) às condições ambientais, da tarefa e do indivíduo ${ }^{13}$.

A intervenção fisioterapêutica promove ao paciente a interação social e a estimulação motora e cognitiva ${ }^{4}$, visto que, em alguns casos de autismo, o padrão de desenvolvimento alterase em comprometimento severo cognitivo, apresentando QI abaixo de 50. Nesses casos, o indivíduo tem maior tendência à autoagressão e dificuldade na linguagem, necessitando de tratamento e acompanhamento por um longo período ${ }^{14}$.

Devido às poucas pesquisas que certifiquem um tratamento específico ao autismo e que comprovem a evolução individual de cada paciente frente às intervenções utilizadas ${ }^{14}$, este estudo tem como objetivo avaliar e promover ganhos nos aspectos afetivo, cognitivo, motor e contato visual nos indivíduos com autismo leve, moderado ou grave, a partir da aplicação da música como auxílio no tratamento fisioterapêutico.

\section{Materiais e métodos}

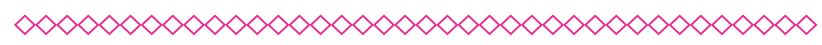

Esta pesquisa caracteriza-se por ser um estudo quanti e qualitativo e realiza-se por meio da análise de relatos de casos, coletados a partir de uma triagem que selecionou indivíduos com o diagnóstico de TEA na Escola de Educação Básica Karin Knebel, na modalidade educação especial, sob entidade mantenedora Nosso Canto (Centro de Adaptação Neurológica Total) do município de Foz do Iguaçu - PR.

Os atendimentos foram realizados na instituição citada, após a aprovação do Comitê de Ética em Pesquisa pela Associação Unificada Paulista de Ensino Renovado Objetivo (Assupero), sob protocolo número 814.526/2014, autorização da instituição proponente e assinatura do Termo de Consentimento Livre e Esclarecido, que autoriza o início do estudo.

0 estudo incluiu três sujeitos do sexo masculino, e os critérios de inclusão surgiram com diagnóstico médico de autismo, a saber: ter entre cinco e sete anos de idade, podendo apresentar grau de autismo leve, moderado ou grave; cabe mencionar que foram excluídos os indivíduos que apresentaram outras complicações neurológicas, deficiência auditiva ou visual e/ou comprometimento motor grave.

As avaliações das crianças ocorreram de duas maneiras, ambas por meio do uso do instrumento de avaliação Childhood Autism Rating Scale

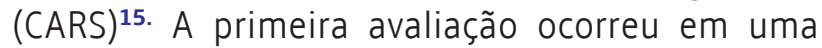
sala de aula, junto com os demais colegas, e foi realizada por uma pedagoga, que avaliava o âmbito social. A outra avaliação ocorreu em uma sala fechada, em que o indivíduo encontrava-se isolado dos demais colegas, e foi realizada por um acadêmico de fisioterapia. O CARS foi utilizado na pré-intervenção (um dia antes do início do primeiro atendimento) e pós-intervenção (um dia após o final do último atendimento) pela pedagoga e pelo acadêmico de fisioterapia.

Esse instrumento de avaliação contempla 15 aspectos a serem analisados na criança com autismo, associando-os à pontuação mínima de um (sem evidência ou anormalidade) e pontuação máxima de quatro (grau severo). A soma dessa pontuação permite a classificação do autismo da seguinte forma: $<30=$ normal; $30-36,5$ = autismo leve a moderado; $>37=$ autismo serevo ${ }^{15}$. Após a avaliação inicial, foi iniciado o protocolo de intervenção, o qual foi elaborado pelos pesquisadores. Durante o estudo, o grupo pesquisado não interrompeu e não alterou suas atividades escolares, terapêuticas e demais afazeres que faziam parte de sua rotina diária.

Como auxílio desse instrumento de avaliação, foram realizadas filmagens durante as intervenções fisioterapêuticas, as quais, respeitando a confidencialidade dos documentos, foram utilizadas apenas para âmbito da pesquisa. As filmagens foram analisadas ao término de todos os atendimentos, por um avaliador independente, sem relação com o paciente que não participou dos outros processos do estudo, a fim de observar 
a evolução qualitativa no comportamento afetivo, contato visual e aprendizado cognitivo e motor do paciente.

No protocolo do estudo, foram realizadas intervenções fisioterapêuticas individuais, três vezes por semana, em um período de dois meses (24 atendimentos por paciente), com quarenta minutos de atendimento cada.

Esses atendimentos eram realizados por dois acadêmicos de fisioterapia, do último ano de formação. Um acadêmico ficou responsável por tocar música ao vivo, utilizando um instrumento musical. Não foi seguido um ritmo musical específico, pois o que foi determinante foi a interação e a atenção do paciente com a música aplicada no período de realizações das tarefas. O segundo acadêmico, junto ao paciente, dava estímulos e possibilitava a compreensão deste na realização das tarefas. Os pacientes foram denominados por $X, Y$ e $Z$ com o intuito de manter o sigilo dos sujeitos.

0 protocolo do estudo foi elaborado devido à escassez bibliográfica direcionada à fisioterapia no indivíduo com autismo e aos efeitos da música e da fisioterapia em um contexto terapêutico. As tarefas (Tabela $\mathbf{1}$ ) basearam-se no aspecto cognitivo, visando o desenvolvimento normal da criança, estimulando o aprendizado motor, a coordenação motora, o alcance, a preensão e a tolerância auditiva, sendo desenvolvidas com o som em conjunto às condutas fisioterapêuticas.

Incluiu-se a utilização de instrumentos sonoros (chocalho infantil e tambor de chocalho infantil) e instrumentos musicais (sanfona infantil, violão e teclado - Casio CTK-50 - com cores e letras especificando cada tecla referente às notas musicais). Juntamente com esses instrumentos, foi utilizado um brinquedo de pelúcia e um brinquedo educativo colorido (Figura 1).

Esse protocolo não seguiu uma sequência pré-estabelecida de intervenção, pois foram respeitadas as necessidades do paciente durante as sessões. Caso o paciente não colaborasse ou não realizasse uma tarefa estabelecida seria proposta a tarefa seguinte.

\section{Resultados}

$\alpha<\infty<\infty<\infty<\infty<\infty<\infty<\infty<\infty<\infty<\infty<\infty<\infty<\infty<\infty<\infty<\infty<\infty$

Na aplicação do CARS na pré-intervenção (Tabela 2), observou-se diferentes graus de autismo entre os indivíduos. Por meio dos vídeos, observaram-se alterações no comportamento afetivo, cognitivo, motor e visual. Para melhor apresentar os resultados, a tabela a seguir (Tabela 3) traz os critérios avaliados separados em três fases do tratamento, sendo a primeira fase correspondente do $1^{\circ}$ ao $4^{\circ} \mathrm{dia}$, a segunda fase do $8^{\circ}$ ao $16^{\circ}$ dia e a terceira fase do $20^{\circ}$ ao $24^{\circ}$ dia de intervenção.

\section{Discussão}

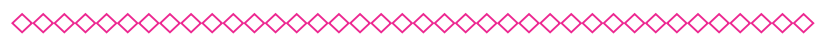

No presente estudo, tendo a música como auxílio na fisioterapia em indivíduos com autismo, notou-se que houve uma evolução no aspecto qualitativo, visando ganhos no comportamento, denotando traços mais definidos de afetividade perante o meio social; na aprendizagem, dando funcionalidade aos objetos com os quais interagiam; e no desenvolvimento motor, apresentando um ganho no controle de tronco e coordenação motora de MMSS. Porém, não foi possivel quantificar essas mudanças por meio da pontuação do CARS (Figura 2). Segundo Lampreia, ${ }^{16}$ para decisões diagnósticas eficazes, o processo de consulta deve incluir os pais, o pedagogo e o médico especialista, pois, em seu estudo, ao aplicar o CARS e outros instrumentos (DSM-IV e PERP-R) observou que a análise qualitativa dos pacientes mostrou competências não reveladas pelos três instrumentos.

A metodologia utilizada nesta pesquisa assemelha-se ao processo musicoterápico evidenciado por Bernardino ${ }^{17}$, que concluiu que a música aumenta a capacidade da criança em manter a atenção concentrada na expressão sonora, possibilitando a ampliação da sua escuta. Visto que, os indivíduos $X$ e $Y$ apresentaram uma evolução considerável na atenção em relacionar o objeto com a nota musical tocada durante a tarefa, aumentando o número de acertos a partir da oitava sessão. 0 paciente $Z$, com autismo 
grave, teve momentos importantes de atenção ao instrumento tocado, mesmo não a mantendo por muito tempo.

Considerando a especificidade apresentada de cada indivíduo em relação ao seu desempenho durante as tarefas, Prestes ${ }^{18}$ identifica ganhos qualitativos no aspecto da tolerância auditiva e atenção. 0 individuo $Z$ passou a identificar o local do estímulo sonoro e se locomover até o esse local, enquanto o individuo $\mathrm{X}$ começou a aceitar tarefas musicais propostas juntamente com os ritmos melódicos, demonstrando uma melhor tolerância auditiva durante as atividades em sala.

Paredes $^{19}$ ainda afirma que a música é importante no desenvolvimento geral das crianças com autismo, pois estimula áreas relevantes do cérebro, como o córtex pré-frontal, córtex prémotor, córtex motor, córtex somatossensorial, lobos temporais, córtex parietal, córtex occipital, cerebelo e áreas do sistema límbico, incluindo a amígdala e 0 tálamo ${ }^{11}$, demonstrando que a complexidade do padrão rítmico ouvido, influencia na atividade neural de quem ouve ${ }^{20}$. Essa observação de Paredes $^{19}$ foi verificada no presente estudo, pois os indivíduos $X$ e $Y$ melhoraram seu desempenho fora do contexto das tarefas propostas, isso foi relatado pela pedagoga que acompanhou as crianças em sala de aula e descreveu que elas compreenderam o ato de abrir a porta e organizar o ambiente de estudo; $\mathrm{o}$ indivíduo $\mathrm{X}$ começou a pronunciar palavras com melhor entendimento e dicção e a linguagem tornou-se mais funcional, apresentando uma evolução no aspecto cognitivo.

Durante as tarefas do protocolo, o indivíduo Y compreendeu os instrumentos sonoros, dando função a eles, perdendo o interesse em levá-los a boca como realizava nas primeiras sessões, deixando também de apontar os objetos a qual se referia, passando a buscá-los conforme o seu desejo. Já o indivíduo Z começou a interagir com os instrumentos sonoros com maior compreensão após a $12^{\circ}$ sessão, deixando assim de realizar movimentos estereotipados em demasia.

Corroborando com estes achados, os estudos de Barros ${ }^{21}$ e Bernardino ${ }^{17}$ constatam que a música desenvolve áreas de comunicação, socialização, psicomotricidade e coordenação motora.
Outro aspecto muito relevante foram os ganhos em relação ao aprendizado motor, nos quais os sujeitos $\mathrm{X}$ e $\mathrm{Y}$ evoluíram bastante, principalmente na manutenção das posturas ajoelhadas e semiajoelhadas, podendo, assim, liberar os membros superiores para tocar os instrumentos sonoros durante a tarefa. Segundo a pedagoga, o paciente $Z$ apresentou evolução significativa na preensão dos objetos por um tempo maior, pois passou a realizar a tarefa de levar sua bandeja até a cozinha, segurar a caneca de água e pegar a colher durante sua alimentação.

Nota-se, assim, queaatividademusicalinfluencia na funcionalidade dos indivíduos submetidos à realização de exercícios associados à música, denotando desenvolvimento na psicomotricidade, traçados mais definidos e precisos, postura correta ao caminhar e equilíbrio postural ${ }^{21,22}$.

Apesar da percepção de ganhos qualitativos consideráveis seguindo o protocolo fisioterapêutico com auxilio da música, identificaram-se características limitantes deste trabalho. Barros ${ }^{21} \mathrm{e}$ Bosa ${ }^{5}$ corroboram comestesachados, afirmando que, aoobservaremindivíduoscomautismo, identificaram aspectos emocionais e comportamentais, como a inflexibilidade e a dificuldade de relacionamento interpessoal. Consequentemente, no presente estudo, ao seguir um protocolo, a mudanças das tarefas desencadeava irritabilidade, rigidez e inflexibilidade, principalmente na observação dos sujeitos Xe Z.

A possível utilização da música no tratamento de distúrbios neurológicos, segundo Rocha e Boggio, ${ }^{11}$ é uma questão que, apesar de apresentar bons indicativos, ainda necessita de maior exploração a fim de chegar a propostas concretas de tratamentos, além disso, para Benavides e Orrego ${ }^{9}$, a linguagem musical deve ser utilizada como uma alternativa comunicativa, baseando-se nos ritmos e percussões, gerando estados de atenção conjunta entre o terapeuta e o paciente com autismo.

\section{Conclusão}

$\infty \times \infty \times \infty \times \infty \times \infty \times \infty \times \infty \times \infty \times \infty \times \infty \times \infty \times \infty \times \infty)$

Com este trabalho conclui-se que a música em conjunto com a fisioterapia pode promover algum 
ganho cognitivo, motor e de interação social em crianças com autismo. Considerando que as evoluções apresentadas quantitativamente foram sutis, ressaltamos a importância de um maior período de intervenções e uso de instrumentos de avaliações mais sensíveis para as características comportamentais individuais nos indivíduos com autismo.

Necessita-se de mais estudos com essa população e de um maior direcionamento nas abordagens, visto as necessidades variáveis apresentadas e aos ganhos qualitativos demostrados neste estudo nos aspectos afetivo, cognitivo, motor e de contato visual.

Para uma abordagem terapêutica mais efetiva na população avaliada, sugere-se a estimulação aliada à integração multidisciplinar, pois ainda não se evidência, na pesquisa, uma abordagem que seja totalmente eficaz para os indivíduos com autismo.

\section{Referências}

$\infty \times \infty \times \infty \times \infty \times \infty \times \infty \times \infty \times \infty \times \infty \times \infty \times \infty \times \infty)$

1. Al-Ayadhi L, Halepoto DM. Role of Proteomics in the Discovery of Autism Biomarkers. J. Coll. of Physicians Surg. Pak. 2013; 23 (2):137-143.

2. Rego SWSE. Autismo: Fisiopatologia e biomarcadores [Dissertação]. Covilhã: Faculdade de Ciências da Saúde, Universidade da Beira Interior, Covilhã; 2012.

3. American Psychiatry Association. DSM-IV Manual diagnóstico e estatístico de transtornos mentais. Porto Alegre: Artes Médicas; 1995.

4. Segura DCA, Nascimento FC, Klein D. Estudo do Conhecimento Clínico dos Profissionais da Fisioterapia no Tratamento de Crianças Autistas. Arq. Ciênc. Saúde. 2011; 15(2):159-165.

5. Bosa CA. As Relações entre Autismo, Comportamento Social e Função Executiva. Psicol. Refl. Crít. 2001; 14 (2):281-287.

6. Wan CY, Ruber T, Hohmann A, Schlaug G. The Therapeutic Effects of Singing in Neurological Disorders. Music Percept. 2010; 27(4):287-295.
7. Brendani H, Paula CS, Bordini D, Rolim D, Portolese J, Pacifico M et al. Autism spectrum disorders: an overview on diagnosis and treatment. Rev. Bras. Psiquiatr. 2009; 35(1):62-72.

8. Hardy MW, Lagasse B. Rhythm, movement, andautism: using rhythmic rehabilitation research as a mode for autism. Front. Integr. Neurosci. 2013; 7(19):1-9.

9. Benavides $H$, Orrego P. La evaluacion del desarrollo de las capacidades de referencia conjunta a través de uma intervención basada em práctica musicales en um niño com transtorno de espectro de autismo. Psicoperspectivas. 2013;9(2):224-252.

10. Corte B, Neto PL. A musicoterapia na doença de Parkinson. Ciênc. Saúde Colet. 2009; 14(6);2295-2304.

11. Rocha VC, Boggio PS. A música por uma óptica neurocientífica. PER MUSI. 2013; (27):132-140.

12. Molnar-Szackacs I, Overy K. Music and mirror neurons: from motion to 'e'motion. Soc. Cogn. Affect. Neurosci. 2006; 1:234-241.

13. Bertoldi AL, Israel VL, Ladewing I. O papel da atenção na fisioterapia neurofuncional. Fisioter. Pesqui. 2011; 18(2): 195-200.

14. Bosa CA. Autismo: intervenções psicoeducacionais. Rev. Bras. Psiquiatr. 2006; 28(Suppl 1): s47-s53.

15. Schopler E, Reichler R, Renner BR. The Childhood Autism Rating Scale (CARS). 10th ed. Los Angeles, CA: Western Psychological Services; 1988.

16. Lampreia C. Avaliações Quantitativa e Qualitativa de um menino autista: uma análise crítica. Psicol. estud. 2003; 8(1):57-65.

17. Bernardino IMFIM. A Música no Desenvolvimento da Comunicação e Socialização da Criança/Jovem com Autismo [Dissertação]. Beja: Escola Superior de Educação, Instituto Politénico de Beja, Beja; 2013.

18. Prestes C. Musicoterapia: estudo de caso de uma criança autista. In: Anais do XVII Encontro Nacional da ABEM: Diversidade musical e 
compromisso social; 8-11 de outubro 2008; São Paulo. São Paulo, ABEM, 2008.

19. Paredes SSG. O papel da Musicoterapia no desenvolvimento cognitivo nas crianças com Perturbação do Espectro do Autismo [Dissertação]. Lisboa: Escola Superior de Educação Almeida Garrett, Lisboa; 2012.

20. Chen JL, Penhume VB, Zatorre RJ. Moving on Time: Brain Network for Auditory-Motor Synchronization is Modulated by Rhythm Complexity and Musical Training. J. Cogn. Neurosci. 2008;20(2):226-239.

21. Barros MRM. A Música como mediadora no desenvolvimento cognitivo em crianças com perturbações Autísticas: Intervenção junto de uma aluna com perturbações Autísticas [Dissertação]. Lisboa, Escola Superior de Educação João de Deus, Lisboa; 2012.

22. Alessandri M, Thorp D, Mundy P, Tuchman RF. Podemos curar el autismo? Del desenlace clínico a la intervención. Rev. Neurol. 2005; 40:131-136. 


\section{Anexos}

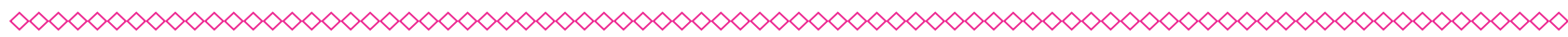

Tabela 1: Protocolo de intervenção

\begin{tabular}{|c|c|}
\hline Tarefa & Objetivo \\
\hline $\begin{array}{l}\text { 1. Paciente sentado, realizar alcance e preensão do } \\
\text { chocalho e tambor de chocalho, acompanhando o } \\
\text { ritmo musical estabelecido pelo acadêmico. }\end{array}$ & $\begin{array}{l}\text { Alcance e preensão; } \\
\text { Controle de tronco; } \\
\text { Percepção sonora; } \\
\text { Coordenação motora fina; } \\
\text { Interação ao meio. }\end{array}$ \\
\hline $\begin{array}{l}\text { 2. Paciente ajoelhado e semiajoelhado, realizar } \\
\text { alcancee preensãodochocalhoetambordechocalho, } \\
\text { acompanhando o ritmo musical estabelecido pelo } \\
\text { acadêmico. }\end{array}$ & $\begin{array}{l}\text { Alcance e preensão; } \\
\text { Controle de tronco; } \\
\text { Troca de postura; } \\
\text { Percepção sensorial; } \\
\text { Noção corporal. }\end{array}$ \\
\hline $\begin{array}{l}\text { 3. Paciente sentado ou semiajoelhado, manusear } \\
\text { a sanfona infantil, acompanhando o ritmo musical } \\
\text { estabelecido. }\end{array}$ & $\begin{array}{l}\text { Criatividade; } \\
\text { Controle de tronco; } \\
\text { Coordenação motora fina e grossa; } \\
\text { Percepção sonora; } \\
\text { Interação ao meio; } \\
\text { Lateralidade; } \\
\text { Contato visual. }\end{array}$ \\
\hline $\begin{array}{l}\text { 4. Paciente ajoelhado, tocar teclado conforme as } \\
\text { cores estabelecidas nas teclas. }\end{array}$ & $\begin{array}{l}\text { Cognição; } \\
\text { Memória; } \\
\text { Coordenação motora fina; } \\
\text { Interação ao meio; } \\
\text { Contato visual. }\end{array}$ \\
\hline $\begin{array}{l}\text { 5. Paciente sentado, distinguir qual objeto refere se } \\
\text { à nota musical tocada pelo acadêmico. }\end{array}$ & $\begin{array}{l}\text { Cognição; } \\
\text { Coordenação motora fina; } \\
\text { Alcance e preensão; } \\
\text { Contato visual; } \\
\text { Percepção sonora. }\end{array}$ \\
\hline $\begin{array}{l}\text { 6. Paciente em pé, realizar alcance e preensão do } \\
\text { objeto no ponto de partida, deambular ao ritmo } \\
\text { da música estabelecido pelo acadêmico e deixar o } \\
\text { objeto no ponto final. }\end{array}$ & $\begin{array}{l}\text { Deambulação; } \\
\text { Transferência de peso; } \\
\text { Alcance e preensão; } \\
\text { Interação ao meio; } \\
\text { Noção corporal; } \\
\text { Percepção sonora. }\end{array}$ \\
\hline
\end{tabular}


Tabela 2: Identificação dos participantes do estudo

\begin{tabular}{|c|c|c|c|c|}
\hline \multirow{2}{*}{ Paciente } & Idade & \multicolumn{2}{|c|}{ CARS pré-intervenção } & \multirow{2}{*}{ Grau de autismo } \\
\cline { 3 - 4 } & & Pedagoga & Fisioterapia & \\
\hline $\mathrm{X}$ & 5 anos & 29,5 & 30,5 & Autismo leve/moderado \\
\hline $\mathrm{Y}$ & 6 anos e 3 meses & 32 & 33 & Autismo leve/moderado \\
\hline $\mathrm{Z}$ & 6 anos e 7 meses & 49 & 51,5 & Autismo grave \\
\hline
\end{tabular}

= Normal: 15 - 29,5; Autismo leve/moderado: 30 - 36,5; Autismo grave: acima 37.

Tabela 3: Análise dos vídeos

\begin{tabular}{|c|c|c|c|c|c|c|c|c|c|c|c|c|c|}
\hline & \multicolumn{2}{|c|}{$\begin{array}{c}\text { Comportamento } \\
\text { afetivo }\end{array}$} & $\begin{array}{c}\text { Aprendizado } \\
\text { Cognitivo }\end{array}$ & \multicolumn{2}{|c|}{$\begin{array}{c}\text { Aprendizado } \\
\text { Motor }\end{array}$} & \multicolumn{3}{|c|}{ Contato Visual } \\
\hline Indivíduo X & M & M & M & P & M & M & P & M & M & A & A & A \\
\hline Indivíduo Y & P & M & P & P & M & M & P & M & M & A & A & A \\
\hline Indivíduo Z & I & P & P & I & P & P & P & P & P & I & P & P \\
\hline
\end{tabular}

I - insatisfatório; P - pobre; M - moderado; A - adequado. 
Figura 1: Exemplos de algumas tarefas do protocolo

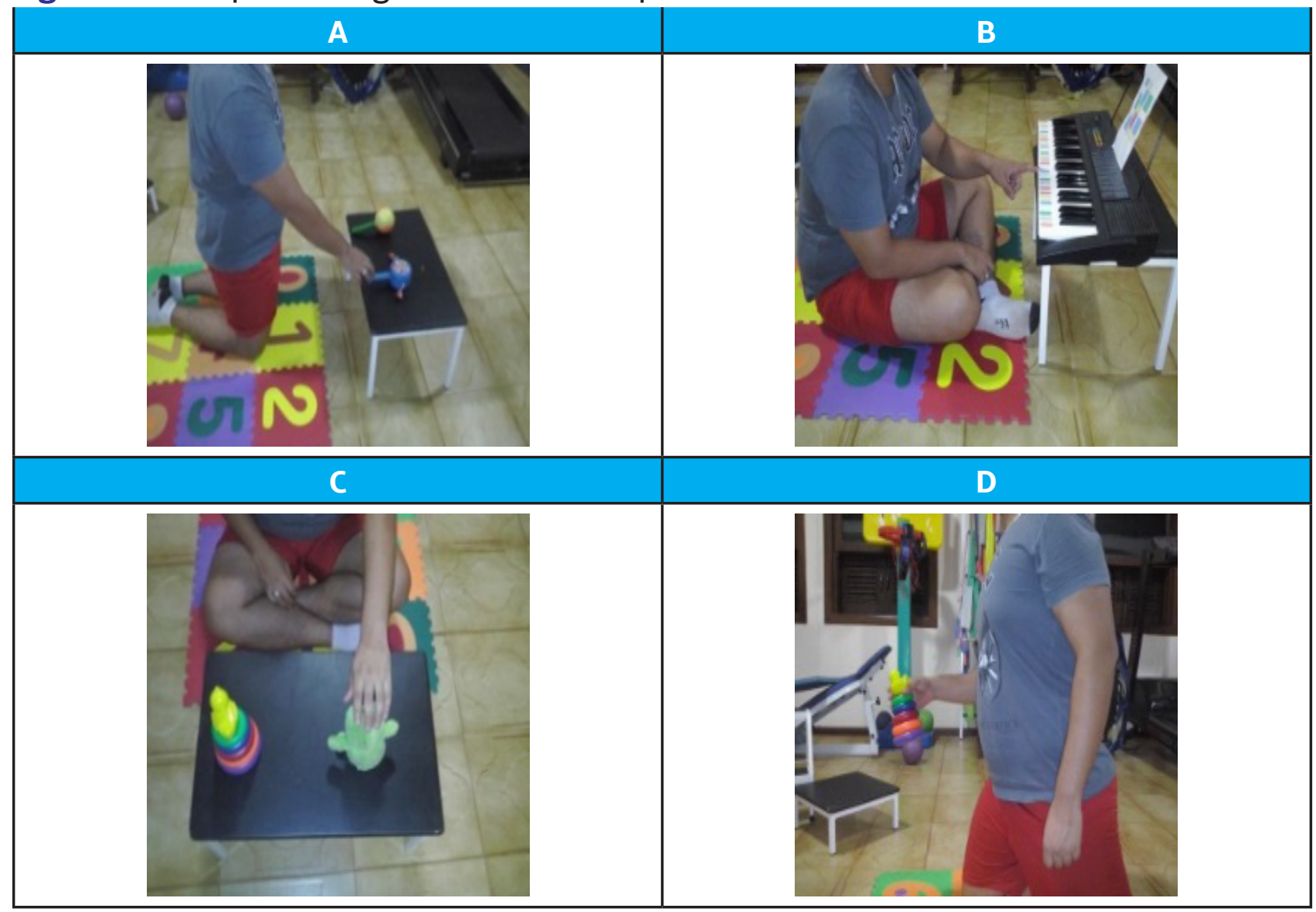

(A) Pegar o objeto sonoro ajoelhado; (B) Tocar o teclado conforme a cor correspondente; (C) Pegar o objeto correspondente à nota musical tocada; (D) Pegar o objeto e leva-lo até outro local

(clique para voltar ao texto) 
Figura 2: CARS pré e pós-intervenção

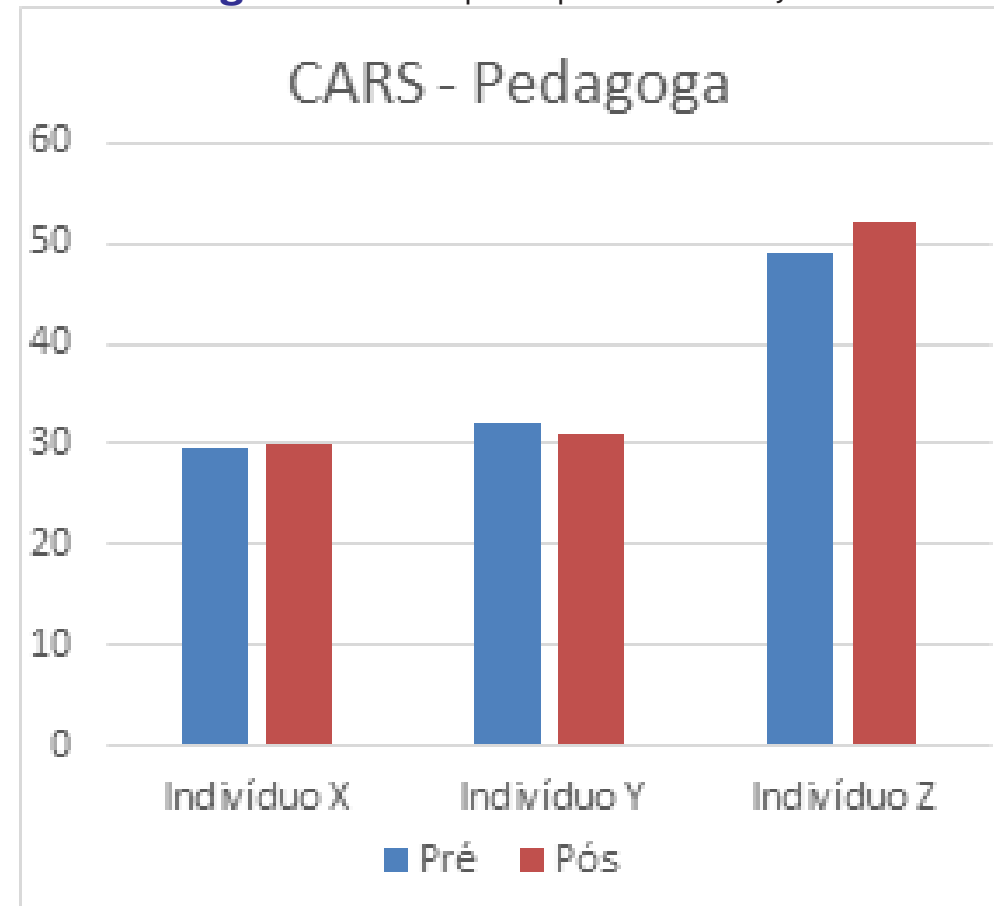

\section{CARS- Fisioterapia}

60

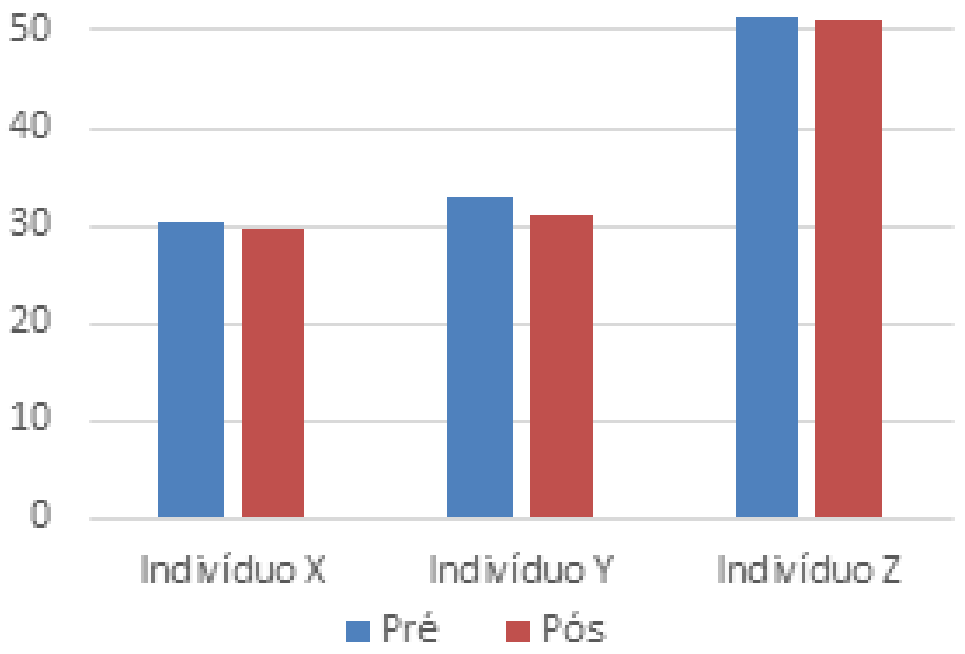

(clique para voltar ao texto) 